\title{
Using Eye Tracking for Interaction
}

\author{
Anneli Olsen \\ Tobii Technology, AB \\ Box 743 \\ 18217 Danderyd, SWEDEN \\ anneli.olsen@tobii.com

\section{Paul Marshall} \\ Warwick Manufacturing Group \\ International Digital Laboratory \\ University of Warwick \\ Coventry, CV4 7AL, UK \\ paul.marshall@warwick.ac.uk
}

\author{
Albrecht Schmidt \\ University of Stuttgart \\ Pfaffenwaldring $5 a$ \\ 70569 Stuttgart, GERMANY \\ albrecht.schmidt@acm.org \\ Veronica Sundstedt \\ School of Computing \\ Blekinge Institute of Technology \\ 37179 Karlskrona, SWEDEN \\ veronica.sundstedt@bth.se
}

Copyright is held by the author/owner(s).

CHI 2011, May 7-12, 2011, Vancouver, BC, Canada.

ACM 978-1-4503-0268-5/11/05.

\begin{abstract}
The development of cheaper eye trackers and open source software for eye tracking and gaze interaction brings the possibility to integrate eye tracking into everyday use devices as well as highly specialized equipment. Apart from providing means for analyzing eye movements, eye tracking also offers the possibility of a natural user interaction modality. Gaze control interfaces are already used within assistive applications for disabled users. However, this novel user interaction possibility comes with its own set of limitations and challenges.
\end{abstract}

The aim of this SIG is to provide a forum for Designers, Researchers and Usability Professionals to discuss the role of eye tracking as a user interaction method in the future as well as the technical and user interaction challenges that using eye tracking as an interaction method brings.

\section{Keywords}

Eye Tracking, Gaze Interaction, Natural User Interface, user interface, user interaction

\section{ACM Classification Keywords}

Interactive systems (H.5.2) User interfaces (D.2.2)

\section{General Terms}

Design, Human factors 


\section{Introduction}

Even though research on the use of eye tracking and gaze control as a user interaction method has been ongoing for over twenty years [1] very few of the results have had much impact in the world outside academia. The only area where eye tracking has for interaction has really taken off in a commercial setting is as a communication aid for disabled people. However, with the introduction of cheaper eye trackers and open source projects for eye control [2] as well as the research done by the COGAIN association [3] there is potential for gaze interaction to reach a much broader set of users. There is already ongoing research on how eye tracking can be used to detect drowsiness in drivers [4]; papers have been published on how to improve the efficiency of mouse use with the aid of eye tracking $[5,6]$; work has investigated using eyetracking to resume an interrupted task [7] and research is looking at whether gaze interaction can be used with mobile phones [8].

Some of the user interaction issues with eye tracking and gaze interaction have been known for some time. The Midas touch problem was discussed in 1991 [9]. Some solutions have been provided [10], but quite often with the assumption that eye tracking should replace other input devices [11]. In the case of disabled users, this may be the case, but for the majority of computer users, eye tracking could instead be a complement to other user interaction techniques such as mouse or keyboard.

In addition to interface manipulation, eye tracking has the potential to be used for more subtle interaction. In recent years, the interest in $3 \mathrm{D}$ and $3 \mathrm{D}$ projections has grown significantly, even outside the academic world, with the introduction of 3D cinemas and 3D TV sets. In order for the human brain to interpret what the eyes see as 3D it needs to receive a slightly different image from each eye [12]. By knowing the eye position of a viewer, advanced 3D projection systems can be constructed. One such system has been patented by Apple [13] and Toshiba demoed a 3D projection capable laptop that did not require glasses due to the use of eye tracking [14] at CES 2011 [15] earlier this year.

$3 \mathrm{D}$ projection is not the only area where eye tracking has shown potential; with the introduction of portable, light weight eye trackers, interest has grown for using eye tracking in augmented reality applications [16].

A more proof of concept-project was developed by Free University of Berlin in corporation with an eye tracker manufacturer in which they steered a car using gaze control [17]. While this project is a fun illustration of what can be done with eye tracking it also highlights that any technology can be potentially dangerous. If the car follows every eye movement, looking at a pedestrian on the sidewalk can have devastating consequences.

As the examples above show, there are a wide variety of applications for which gaze interaction can be used. However, there are still many challenges to be addressed before gaze interaction can be successfully integrated into the everyday lives of regular people, or even in specialized equipment for experts. In this SIG we aim to facilitate a discussion about how eye tracking can be used for interaction. By bringing researchers, designers and usability professionals together we want to explore further possibilities for gaze interaction as 
well as identify the key technical and user interaction challenges and potential solutions for these regarding using eye tracking for interaction. Our goal is to start a discussion that results in future development of theories, solutions, ideas, frameworks and recommendations as well as practical considerations about the use of gaze detection as a mode of interaction.

\section{Audience}

This SIG is for anyone who is interested in eye tracking used for human computer interaction. Gaze interaction can be used in many different areas ranging from regular human computer interaction to augmented reality as well as interaction between humans and cars or other physical devices. Hence, we would value participation from researchers or practitioners within every possible or even (currently) impossible field where eye tracking could be used. Potential groups of participants who would benefit from participating in this SIG could be, but are not limited to, researchers, designers and technologist interested in or who are currently developing:

- Multimodal interaction interfaces.

- 'Natural' user interfaces.

- Interaction for disabled users.

- Systems where using hands for interaction is not an option, e.g., medical devices.

- Systems regarding safety, such as drowsiness prevention, risk assessment, risk detection etc.

- Augmented reality displays and interfaces.

- 3D projection interfaces.

\section{SIG Organization}

The SIG will be organized as follows:

- Presentation of the purpose of the SIG and the organizers (5 $\mathrm{min}$ )

- Introductions by the participants (10 $\mathrm{min}$ )

- Demo and presentation of how eye tracking is and can be used for interaction (10 $\mathrm{min}$ )

- Interactive discussion about interaction methods available when using gaze interaction. Identification of the interaction principles and challenges associated with these methods and possible solutions ( $25 \mathrm{~min}$ )

- Interactive discussion about technical challenges when using eye tracking as an interaction method. Where are the limitations, what implications do these limitations have for the interaction and what can be done to compensate for these limitations. (20 min)

\section{References}

[1] Majaranta, P., and Räihä, K-J. Twenty years of eye typing: systems and design issues. In Proc. ETRA '02, ACM Press (2002) 15-22.

[2] DFKI German Research Center for Artificial Intelligence. Text 2.0 - What if your book really knew where you are gazing at? [Online]. http://text20.net/

[3] COGAIN. Communiation by Gaze Interation. [Online]. http://www.cogain.org/association

[4] Buciu, I., Gacsádi, A., and Grava, C. Vision based Approaches for Driver Assistance Systems. In Proc. ICAI'10, WSEAS, (2010) 92-97.

[5] Monden, A., Matsumoto, K. and Yamato, M. Evaluation of gaze-added target selection methods suitable for general GUIs. International Journal of Computer Applications in Technology, 24, 1, (2005) 1724. 
[6] Blanch, R. and Ortega, M. Rake cursor: improving pointing performance with concurrent input channels.

In Proc. CHI '09, ACM Press (2009) 1415-1418.

[7] Kern, D., Marshall, P., and Schmidt, A. Gazemarks - Gaze-Based Visual Placeholders to Ease Attention Switching. In CHI '10, (2010) 2093-2102.

[8] Miluzzo, E., Wang, T., and Campbell, A.T. EyePhone: Activating Mobile Phones With Your Eyes. In Proc. MobiHeld '10, ACM Press, (2010) 15-20.

[9] Jacob, R.J.K. The use of eye movements in humancomputer interaction techniques: what you look at is what you get. ACM Transactions on Information

Systems (TOIS) - Special issue on computer-human interaction, 9, 2, (1991) 152-169.

[10] Istance, H., Bates, R., Hyrskykari, A., and Vickers, S. Snap clutch, a moded approach to solving the Midas touch problem. In Proc. ETRA '08, ACM Press, (2008) 221-228.

[11] Huckauf, A., Goettel, T., Heinbockel, M., and

Urbina, M. What you don't look at is what you get: anti- saccades can reduce the midas touch-problem. In Proc APGV '05, ACM Press, (2005) 170.

[12] Green, P.R., Georgeson, M.A., and Bruce, V. Visual perception: physiology, psychology, and ecology. New York, NY: Psychology Press, 2003.

[13] Krah, C.H. Three-dimensional display system.

7,843,449, September 20, 2006.

[14] Mokey, N. (2011, January) Digital trends. [Online]. http://www digitaltrends.com/trade-

shows/ces/toshibas-3d-laptop-uses-webcam-to-trackeyes-eliminates-glasses/

[15] (2011) 2011 International CES. [Online].

http://www.cesweb.org/

[16] Baldauf, M., Fröhlich, P., and Hutter, S. KIBITZER: a wearable system for eye-gaze-based mobile urban exploration. In Proc. AH '10, ACM Press, (2010) 1-5.

[17] (2010) Freie Universität Berlin: Office of News and Public Affairs. [Online]. http://www.fu-

berlin.de/en/presse/fup/2010/fup 10 106/index.html 